\title{
Tracoma: estudo epidemiológico de escolares em Alagoas - Brasil
}

\author{
Trachoma: epidemiologic study of scholars from Alagoas State - Brazil
}

\author{
Renato Wendell Ferreira Damasceno ${ }^{1}$ \\ Rodrigo RibeiroSantos ${ }^{2}$ \\ Thiago Rodrig'ues Tavares Cavalcanti ${ }^{3}$ \\ Richard Yudi Hida ${ }^{4}$ \\ MárioJorge Santos ${ }^{5}$ \\ Andréa Maria Cavalcante Santos ${ }^{6}$ \\ Paulo Elias Correa Dantas ${ }^{7}$
}

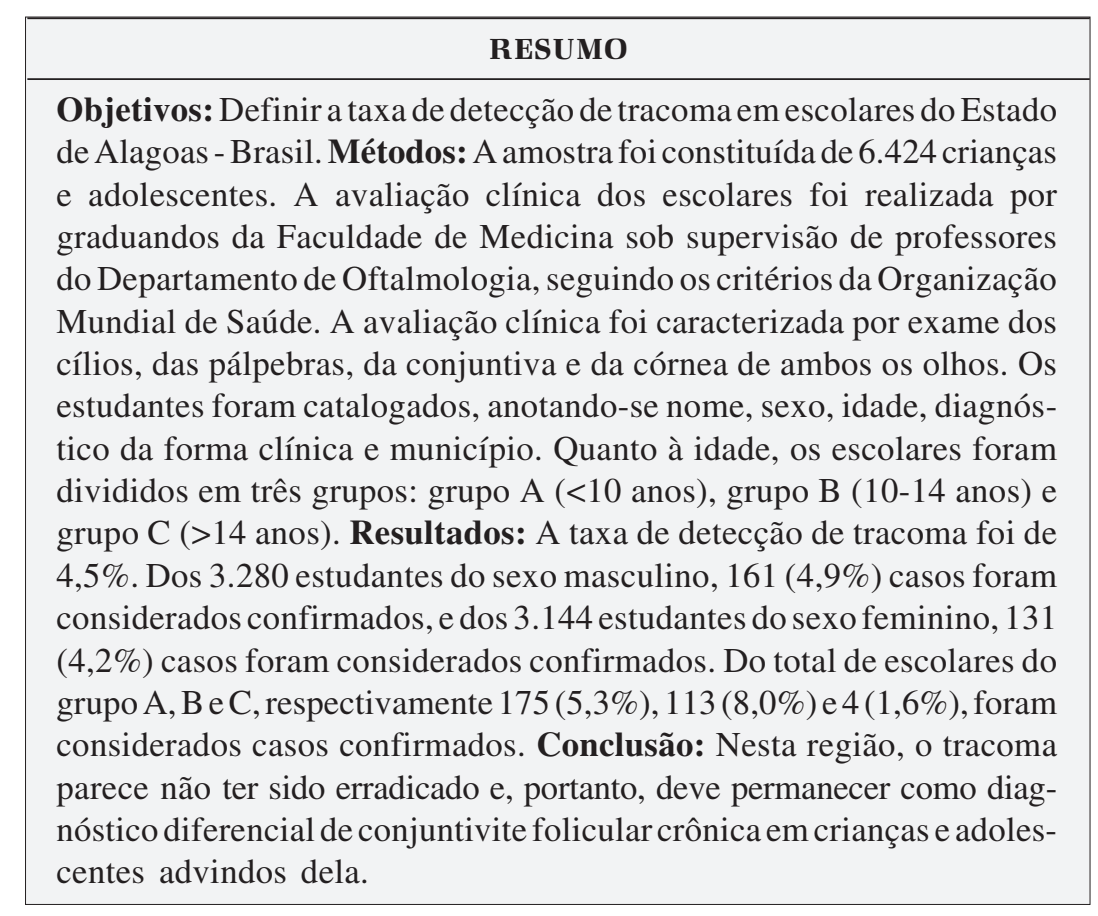

Descritores: Tracoma/epidemiologia; Tracoma/diagnóstico; Conjuntivite/diagnóstico; Córnea; Entrópio; Saúde escolar; Estudantes/estatística \& dados numéricos

da Faculdade de Medicina da Universidade Federal de Alagoas - UFAL - Maceió (AL) - Brasil.

${ }^{1}$ Pós-graduando (Doutorado) do Departamento de Oftalmologia da Universidade Federal de São Paulo UNIFESP - São Paulo (SP) - Brasil.

${ }^{2}$ Estagiário do Setor de Córnea do Departamento de Oftalmologia da Faculdade de Ciências Médicas da Santa Casa de São Paulo - FCMSCSP - São Paulo (SP) - Brasil.

${ }^{3}$ Residente do Departamento de Radiologia do Hospital Heliópolis - São Paulo (SP) - Brasil.

${ }^{4}$ Médico Assistente do Setor de Córnea da FCMSCSP - São Paulo (SP) - Brasil.

${ }_{5}^{5}$ Professor da Faculdade de Medicina da UFAL - Maceió (AL) - Brasil.

${ }^{6}$ Responsável pelo Setor de Córnea do Departamento de Oftalmologia da Faculdade de Medicina da UFAL-Maceió (AL) - Brasil.

Professor do Departamento de Oftalmologia da FCMSCSP - São Paulo (SP) - Brasil.

Endereço para correspondência: Renato Wendell F. Damasceno. Rua Jaguaribe, 102/113 - São Paulo (SP) CEP 01224-000

E-mail: renatowendell@hotmail.com

Recebido para publicação em 29.08.2008

Última versão recebida em 10.03.2009

Aprovação em 23.03.2009

\section{INTRODUÇÃO}

O tracoma é afecção inflamatória ocular crônica, cujo agente etiológico é a Chlamydia trachomatis (sorotipos A, B, Ba e C), uma bactéria gramnegativa, que produz uma ceratoconjuntivite crônica recidivante e transmissível ${ }^{(1-3)}$. A única fonte de infecção é o homem com infecção ativa na conjuntiva ou em outras mucosas ${ }^{(2-4)}$. As formas de transmissão são a direta, de olho a olho, ou a indireta, através de objetos contaminados ${ }^{(2-4)}$. Alguns insetos, como a mosca doméstica (Musca domestica) podem atuar como vetores $^{(1)}$. Em decorrência de infecções repetidas, o tracoma produz cicatrizes na conjuntiva palpebral, podendo levar à formação de entrópio e triquíase $^{(5-6)}$. Estas podem levar a alterações irreversíveis da córnea, causando cegueira $^{(7)}$.

No Brasil, o tracoma foi encontrado inicialmente no Nordeste a partir do século XVIII, constituindo-se o primeiro foco de tracoma no país ${ }^{(1)}$. Além deste, outros dois focos teriam contribuído decisivamente para a disseminação do tracoma no Brasil: Rio Grande do Sul e São Paulo, ambos iniciados com a intensificação da imigração européia ${ }^{(1)}$.
} 
A prevalência do tracoma sempre esteve relacionada ao baixo índice socioeconômico e à falta de saneamento bási$\mathrm{co}^{(3-6,8-14)}$. Mais especificamente, no Brasil, com o ciclo de desenvolvimento econômico na década de 60 , verificou-se uma diminuição acentuada do número de casos de tracoma, o que culminou com a conclusão errônea de que a doença havia sido erradicada $^{(1)}$. Assim, durante as últimas décadas, o diagnóstico deixou de ser feito por falta de capacitação dos médicos generalistas e oftalmologistas, devido à falta de contato com pacientes com tracoma ou ao erro no diagnóstico ${ }^{(1)}$.

Diversos estudos demonstraram a ocorrência de tracoma na maior parte do Brasil, sendo encontrados casos em todas as regiões e em diversas comunidades, tanto em grandes centros urbanos como em áreas rurais, cidades litorâneas ou amazônicas, assim como em comunidades indígenas $\operatorname{afastadas}^{(15-23)}$.

Há uma perspectiva da Organização Mundial de Saúde de erradicação do tracoma até o ano de 2020 com o programa chamado "SAFE 2020", representado pelo combate à transmissão do tracoma e suas consequências: Surgery (cirurgia para correção de triquíase), Antibiotics (antibióticos para tratamento), Facial Cleanliness (limpeza das mãos e do rosto) e Environmental Improvement (melhoria ambiental) $)^{(1,6,24-26)}$.

Devido às características socioeconômicas peculiares da região, definimos como objetivo deste estudo, prospectar e definir qual a taxa de detecção de tracoma em escolares do Estado de Alagoas via inquérito epidemiológico.

\section{MÉTODOS}

Este é um estudo transversal e observacional realizado pelo Departamento de Oftalmologia da Faculdade de Medicina da Universidade Federal de Alagoas em 2004. Este estudo foi aprovado pelo Comitê de Ética em Pesquisa da Universidade Federal de Alagoas. Foi realizado um inquérito epidemiológico em amostra de escolares da rede pública de ensino nos municípios do Estado de Alagoas.

\section{Tamanho da amostra}

O tamanho da amostra foi determinado, considerando-se uma prevalência média de $5 \%$ de tracoma em todas as suas formas clínicas, aceitando-se um erro máximo de amostragem de $0,01 \mathrm{em} 95 \%$ das possíveis amostras ${ }^{(20)}$. A amostra foi constituída de 6.424 crianças e adolescentes selecionados previamente por amostragem por conglomerados.

\section{Amostragem}

A amostragem foi realizada a partir de escolares da rede pública de ensino do Estado de Alagoas, utilizando o Censo de Escolares do Ministério da Educação de 2003. Todos os municípios do Estado de Alagoas com índice de desenvolvimento humano (IDH) menor que a média nacional de $2003(0,792)$ fizeram parte da população de estudo, constituindo-se um grupo de 51 municípios, representando as três mesorregiões do Estado de Alagoas: Sertão, Agreste e Leste (Figura 1).

Foi realizado cálculo da média de alunos por escola para definir o tamanho adequado das unidades de amostragem. O sorteio foi realizado iniciando-se com um número aleatório e somando-se o intervalo de amostragem.

\section{Diagnóstico de tracoma}

Para diagnosticar os casos de tracoma, a avaliação clínica dos escolares foi realizada por graduandos da Faculdade de Medicina sob supervisão de professores do Departamento de Oftalmologia da Faculdade de Medicina da Universidade Federal de Alagoas, seguindo os critérios da Organização Mundial de Saúde ${ }^{(1)}$. Os examinadores foram previamente treinados e capacitados por representantes da Secretaria de Saúde do Estado de Alagoas.

A avaliação clínica foi caracterizada por exame dos cílios, das pálpebras, da conjuntiva e da córnea de ambos os olhos com a iluminação adequada e com o auxílio de lupa de $4 \mathrm{x}$ de magnificação. Foi considerado caso confirmado de tracoma, qualquer paciente cujo exame oftalmológico apresentou um ou mais dos seguintes sinais ${ }^{(1-2)}$ :

- Inflamação tracomatosa folicular (TF): Cinco ou mais folículos de no mínimo $0,5 \mathrm{~mm}$ de diâmetro na conjuntiva tarsal superior;

- Inflamação tracomatosa intensa (TI): Espessamento inflamatório da conjuntiva tarsal superior, não permitindo a observação de mais da metade dos vasos tarsais profundos;

- Cicatrização conjuntival tracomatosa (TS): Conjuntiva tarsal superior com aparência fibrosa, esbranquiçada e com bordas retas, angulares ou estreladas;

- Triquíase tracomatosa (TT): Um ou mais cílios atritando a superfície ocular ou evidência de remoção recente de cílios invertidos associados a cicatrizes na conjuntiva tarsal superior sugestivas de tracoma;

- Opacificação corneana (CO): Opacificação da córnea de fácil observação na área central.

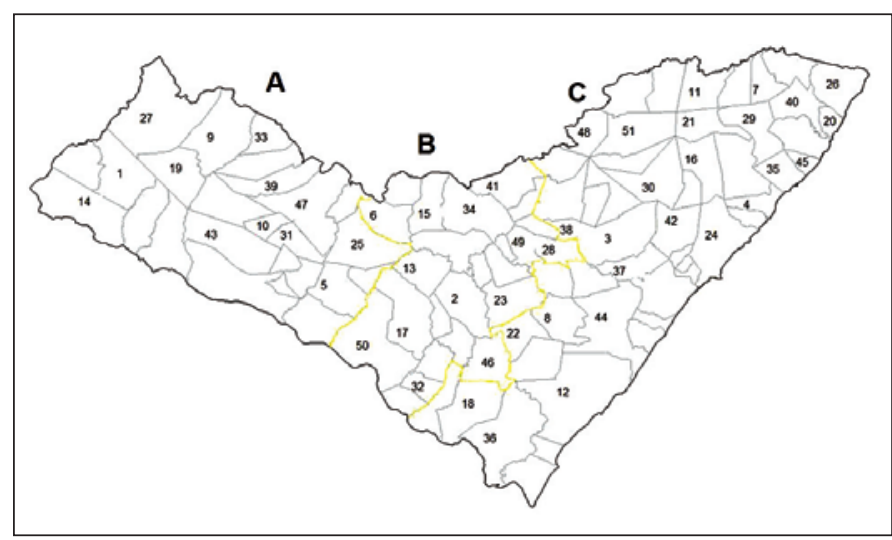

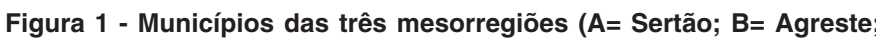
C= Leste) do Estado de Alagoas participantes do estudo epidemiológico de tracoma 


\begin{tabular}{|c|c|c|c|c|c|c|c|}
\hline Sexo & GE & TF (\%) & TI (\%) & TS (\%) & TT (\%) & CO (\%) & Total (\%) \\
\hline \multirow{3}{*}{ Masculino } & A & $97(2,9)$ & 0 & 0 & 0 & 0 & $97(2,9)$ \\
\hline & B & $62(1,9)$ & 0 & 0 & 0 & 0 & $62(1,9)$ \\
\hline & C & $2(0,1)$ & 0 & 0 & 0 & 0 & $2(0,1)$ \\
\hline \multirow[t]{2}{*}{ Total } & - & $161(4,9)$ & 0 & 0 & 0 & 0 & $161(4,9)$ \\
\hline & A & $78(2,5)$ & 0 & 0 & 0 & 0 & $78(2,5)$ \\
\hline \multirow[t]{2}{*}{ Feminino } & B & $51(1,6)$ & 0 & 0 & 0 & 0 & $51(1,6)$ \\
\hline & $\mathrm{C}$ & $2(0,1)$ & 0 & 0 & 0 & 0 & $2(0,1)$ \\
\hline Total & - & $131(4,2)$ & 0 & 0 & 0 & 0 & $131(4,2)$ \\
\hline
\end{tabular}

Após a identificação dos casos positivos, foi calculada a taxa de detecção de tracoma para cada município de acordo com a fórmula fornecida pelo Ministério da Saúde (Taxa de detecção $=\mathrm{N}^{\mathrm{o}}$ de $\operatorname{casos}$ positivos $\mathrm{x} 100 / \mathrm{N}^{\mathrm{o}}$ total de examinados) ${ }^{(1)}$.

Os estudantes foram catalogados, anotando-se nome, sexo, idade, diagnóstico da forma clínica e município. Quanto à idade, os escolares foram divididos em três grupos: grupo $\mathrm{A}$ (escolares menores que 10 anos), grupo B (escolares de 10-14 anos) e grupo $\mathrm{C}$ (escolares maiores que 14 anos).

Concomitantemente, foram realizadas atividades educacionais sobre tracoma, enfatizando o diagnóstico, a prevenção e o controle com escolares, familiares e professores. Os casos de inflamação tracomatosa folicular e inflamação tracomatosa intensa diagnosticados foram tratados com azitromicina $20 \mathrm{mg} / \mathrm{kg}$ de peso (dose máxima de $1 \mathrm{~g}$ ) em dose única por via oral e referidos para acompanhamento oftalmológico ${ }^{(27-30)}$.

\section{RESULTADOS}

O tracoma foi diagnosticado em 292 escolares de uma amostra total de 6.424 examinados, sendo a taxa de detecção de $4,5 \%$.

Dos 6.424 estudantes examinados, $3.280(51,1 \%)$ foram do sexo masculino e $3.144(48,9 \%)$ foram do sexo feminino. Dos 3.280 escolares do sexo masculino, $161(4,9 \%)$ casos foram considerados confirmados, sendo todos de inflamação tracomatosa folicular (Tabela 1). Dos 3.144 estudantes do sexo feminino, $131(4,2 \%)$ casos foram considerados confirmados, sendo todos de inflamação tracomatosa folicular (Tabela 1).

Dos 6.424 estudantes examinados, $3.329(51,8 \%)$ integraram o grupo A, 2.841 (44,2\%) formaram o grupo B e 254 (4\%) compuseram o grupo C. Do total de escolares do grupo A, $175(5,3 \%)$ foram considerados casos confirmados, sendo todos de inflamação tracomatosa folicular (Tabela 2). Do total de escolares do grupo B, $113(8 \%)$ foram considerados casos confirmados, todos de inflamação tracomatosa folicular (Tabela 2). Do total de escolares do grupo C, 4 (1,6\%) foram considerados casos confirmados, sendo todos de inflamação tracomatosa folicular (Tabela 2).

Com relação à taxa de detecção de cada município, foi evidenciada uma variação de zero (Flexeiras, Japaratinga, Joaquim Gomes e Maribondo) a 20,7\% (Junqueiro), sem relação com a mesorregião (Tabela 3 ).

\section{DISCUSSÃO}

Referências à ocorrência de tracoma foram encontradas desde os primeiros registros humanos, em diferentes civilizações, tais como: China (século XXVII a.C.), Suméria (século XXI a.C.), Egito (século XIX a.C.), Grécia (século V a.C.) e Roma (século I a.C.) $)^{(1-2)}$.

O tracoma é hoje uma das afecções que mais preocupa a Organização Mundial de Saúde, por ser encontrado em grandes proporções em todo o mundo e por suas consequências ${ }^{(2,5)}$. A importância mundial dessa afecção pode ser representada pelos números: é responsável por aproximadamente $3 \%$ dos casos de cegueira em todo o mundo, há cerca de oito milhões de pessoas com cegueira irreversível por tracoma e há uma estimativa de oitenta e quatro milhões de casos de tracoma necessitando de tratamento ${ }^{(1)}$.

A falta de dados oficiais sobre a prevalência do tracoma em Alagoas foi o principal motivo para a realização deste estudo com a população escolar regularmente matriculada em instituições de ensino público distribuídas nos 51 municípios de Alagoas com IDH abaixo da média nacional de 2003. Por meio deste estudo, foi possível realizar concomitantemente um trabalho de prevenção e conscientização em um grande número de escolares e seus familiares.

Os dados deste estudo epidemiológico denotam uma taxa de detecção de tracoma de 4,5\%. A taxa de detecção encontrada em Alagoas encontra-se dentro da faixa verificada em outros Estados, como: Rio Grande do Norte $(3,61 \%)$, Bahia (3,58\%), Paraíba $(3,81 \%)$, Roraima $(4,34 \%)$ e São Paulo $(4,11 \%)^{(1)}$. Diferentemente, alguns outros Estados apresentaram taxas mais

Tabela 2. Distribuição de casos positivos por grupo etário e forma clínica

\begin{tabular}{|c|c|c|c|c|c|c|}
\hline GE & TF (\%) & TI (\%) & TS (\%) & TT (\%) & CO (\%) & Total (\%) \\
\hline$A$ & $175(5,3)$ & 0 & 0 & 0 & 0 & $175(5,3)$ \\
\hline B & $113(8,0)$ & 0 & 0 & 0 & 0 & $113(8,0)$ \\
\hline C & $4(1,6)$ & 0 & 0 & 0 & 0 & $4(1,6)$ \\
\hline \multicolumn{7}{|c|}{$\begin{array}{l}\mathrm{GE}=\text { grupo etário; } \mathrm{A}=\text { escolares menores que } 10 \text { anos; } \mathrm{B}=\text { escolares de } 10-14 \\
\text { anos; } \mathrm{C}=\text { escolares maiores que } 14 \text { anos; } \mathrm{TF}=\text { inflamação tracomatosa folicular; } \\
\mathrm{Tl}=\text { inflamação tracomatosa intensa; } \mathrm{TS}=\text { cicatrização conjuntival tracomatosa; } \\
\mathrm{TT}=\text { triquíase tracomatosa; } \mathrm{CO}=\text { opacificação da córnea }\end{array}$} \\
\hline
\end{tabular}




\begin{tabular}{|c|c|c|c|c|c|c|c|c|c|}
\hline$\#$ & Município & CP & TD & Total & \# & Município & CP & TD & Total \\
\hline 1 & Água Branca & 7 & $7,4 \%$ & 94 & 27 & Mata Grande & 6 & $4,2 \%$ & 143 \\
\hline 2 & Arapiraca & 17 & $5,3 \%$ & 322 & 28 & Maribondo & 0 & 0 & 62 \\
\hline 3 & Atalaia & 4 & $2,4 \%$ & 170 & 29 & Matriz de Camaragibe & 1 & $1,0 \%$ & 98 \\
\hline 4 & Barra S. Antonio & 3 & $2,7 \%$ & 112 & 30 & Murici & 10 & $15,9 \%$ & 63 \\
\hline 5 & Batalha & 1 & $1,8 \%$ & 55 & 31 & Olho D'agua das Flores & 5 & $6,9 \%$ & 72 \\
\hline 6 & Cacimbinhas & 5 & $4,5 \%$ & 110 & 32 & Olho D'agua Grande & 1 & $2,5 \%$ & 40 \\
\hline 7 & Campestre & 1 & $0,7 \%$ & 137 & 33 & Ouro Branco & 1 & $0,9 \%$ & 113 \\
\hline 8 & Campo Alegre & 7 & $5,8 \%$ & 120 & 34 & Palmeira dos Índios & 9 & $10,7 \%$ & 84 \\
\hline 9 & Canapi & 3 & $3,9 \%$ & 77 & 35 & Passo de Camaragibe & 5 & $5,0 \%$ & 99 \\
\hline 10 & Carneiros & 6 & $5,7 \%$ & 105 & 36 & Penedo & 21 & $15,2 \%$ & 138 \\
\hline 11 & Colônia Leopoldina & 11 & $4,7 \%$ & 234 & 37 & Pilar & 2 & $2,1 \%$ & 96 \\
\hline 12 & Coruripe & 21 & $13,3 \%$ & 158 & 38 & Pindoba & 1 & $1,7 \%$ & 60 \\
\hline 13 & Craíbas & 4 & $6,9 \%$ & 58 & 39 & Poço das Trincheiras & 9 & $11,2 \%$ & 80 \\
\hline 14 & Delmiro Gouveia & 6 & $4,8 \%$ & 124 & 40 & Porto Calvo & 5 & $5,0 \%$ & 100 \\
\hline 15 & Estrela de Alagoas & 4 & $4,0 \%$ & 100 & 41 & Quebrângulo & 2 & $5,7 \%$ & 35 \\
\hline 16 & Flexeiras & 0 & 0 & 23 & 42 & Rio Largo & 5 & $6,6 \%$ & 76 \\
\hline 17 & Girau do Ponciano & 4 & $4,4 \%$ & 90 & 43 & São José da Tapera & 13 & $13,7 \%$ & 95 \\
\hline 18 & Igreja Nova & 10 & $8,5 \%$ & 117 & 44 & São Miguel dos Campos & 6 & $6,7 \%$ & 89 \\
\hline 19 & Inhapi & 8 & $10,9 \%$ & 73 & 45 & São Miguel dos Milagres & 2 & $3,8 \%$ & 53 \\
\hline 20 & Japaratinga & 0 & 0 & 34 & 46 & São Sebastião & 1 & $1,0 \%$ & 99 \\
\hline 21 & Joaquim Gomes & 0 & 0 & 121 & 47 & Santana do Ipanema & 1 & $0,9 \%$ & 111 \\
\hline 22 & Junqueiro & 17 & $20,7 \%$ & 82 & 48 & Santana do Mundaú & 1 & $1,3 \%$ & 70 \\
\hline 23 & Limoeiro de Anadia & 4 & $4,1 \%$ & 97 & 49 & Tanque D'arca & 2 & $1,7 \%$ & 117 \\
\hline 24 & Maceió & 26 & $2,1 \%$ & 1.262 & 50 & Traipu & 7 & $9,8 \%$ & 71 \\
\hline 25 & Major Isidoro & 4 & $5,8 \%$ & 69 & 51 & União dos Palmares & 1 & $0,3 \%$ & 310 \\
\hline 26 & Maragogi & 2 & $2,1 \%$ & 95 & & & & & \\
\hline \multicolumn{10}{|c|}{$\mathrm{CP}=$ caso positivo; $\mathrm{TD}=$ taxa de detecção } \\
\hline
\end{tabular}

elevadas, como: Rio Grande do Sul (4,60\%), Tocantins (5,33\%), Sergipe (5,84\%), Paraná (6,2\%), Espírito Santo (4,74\%), Ceará $(7,81 \%)$ e Acre $(8,34 \%)^{(1)}$.

Esta taxa de detecção é alta quando comparada aos resultados de outros estudos na mesma faixa etária: São Paulo - SP $(2,2 \%)^{(15)}$, Presidente Prudente - SP $(2,6 \%)^{(19)}$, Franco da Rocha - SP $(1,5 \%)^{(20)}$ e Francisco Morato - SP $(3,6 \%)^{(20)}$. Entretanto, essa mesma taxa de detecção é mais baixa do que outras encontradas nos municípios de Joinville - SC $(4,9 \%)^{(21)}$ e Manaus - AM $(4,8 \%)^{(22)}$.

Para que seja possível pensar na erradicação mundial do tracoma, faz-se necessária a implantação de programas de saneamento básico em áreas menos favorecidas, como em Alagoas, que detém IDH comparáveis a países pobres da África.

Em resumo, a taxa de detecção de tracoma em escolares no Estado de Alagoas foi de 4,5\%. Nesta região, o tracoma não foi erradicado e deve permanecer no diagnóstico diferencial de conjuntivite folicular crônica em crianças e adolescentes.

\section{ABSTRACT}

Purpose: To define the detection rate of trachoma in scholars from Alagoas - Brazil. Methods: The sample included 6,424 children and adolescents. Scholar's clinical evaluation was performed by medical students under the supervision of pro- fessors of the Department of Ophthalmology, according to the World Health Organization guidelines. The clinical evaluation was characterized by eyelashes, eyelids, conjunctiva and cornea examination in both eyes. The scholars were cataloged and data of name, sex, age, clinical forms diagnosis and city were recorded. Regarding the age, the students were divided into three groups: group A ( $<10$ years old), group B (10-14 years old) and group C ( $>14$ years old). Results: The detection rate of trachoma was $4.5 \%$. From 3,280 male scholars, 161 (4.9\%) cases were confirmed; from 3,144 female scholars, 131 (4.2\%) cases were considered confirmed. From the total scholars in group A, $\mathrm{B}$ and C; respectively 175 (5.3\%), $113(8.0 \%)$ and $4(1.6 \%)$ were considered confirmed cases. Conclusion: In this region, it seems that trachoma has not been eradicated and, therefore, must remain as a differential diagnosis of chronic follicular conjunctivitis in children and adolescents from this region.

Keywords: Trachoma/epidemiology; Trachoma/diagnosis; Prevalence; Conjunctivitis/diagnosis; Cornea; Entropion; School health; Students/statistics \& numerical data

\section{REFERÊNCIAS}

1. Barros OM. Manual de Controle do tracoma. Brasília (DF): Assessoria de Comunicação e Educação em Saúde, 2001. 
2. Wright HR, Turner A, Taylor HR. Trachoma. Lancet. 2008;371(9628):1945-54.

3. Cumberland P, Edwards T, Hailu G, Harding-Esch E, Andreasen A, Mabey D et al. The impact of community level treatment and preventative interventions on trachoma prevalence in rural Ethiopia. Int J Epidemiol. 2008;37(3):549-58.

4. Mabey D. Trachoma: recent developments. Adv Exp Med Biol 2008;609:98-107.

5. Burton MJ. Trachoma: an overview. Br Med Bull. 2007;84:99-116.

6. Cook JA. Eliminating blinding trachoma. N Engl J Med. 2008;358(17):1777-9.

7. Smith AG, Broman AT, Alemayehu W, Munoz BE, West SK, Gower EW. Relationship between trachoma and chronic and acute malnutrition in children in rural Ethiopia. J Trop Pediatr. 2007;53(5):308-12.

8. Khandekar R, Mohammed AJ. The prevalence of trachomatous trichiasis in Oman (Oman eye study 2005). Ophthalmic Epidemiol. 2007;14(5):267-72.

9. Sloan FA, Belsky DW, Boly IA. Prevalence of major eye diseases among US Civil War veterans, 1890-1910. Arch Ophthalmol. 2008;126(2):246-50.

10. Nishimura S. Promoting health during the American occupation of Japan the public health section, Kyoto Military Government Team, 1945-1949. Am J Public Health. 2008;98(3):424-34.

11. Rutzen AR, Ellish NJ, Schwab L, Graham PJ, Pizzarello LD, Hemady RK et al. Blindness and eye disease in Cambodia. Ophthalmic Epidemiol. 2007; 14(6):360-6.

12. Jansen E, Baltussen RM, van Doorslaer E, Ngirwamungu E, Nguyen MP, Kilima PM. An eye for inequality: how trachoma relates to poverty in Tanzania and Vietnam. Ophthalmic Epidemiol. 2007;14(5):278-87.

13. Lansingh VC, Carter MJ. Trachoma surveys 2000-2005: results, recent advances in methodology, and factors affecting the determination of prevalence. Surv Ophthalmol. 2007;52(5):535-46.

14. Ngondi J, Matthews F, Reacher M, Onsarigo A, Matende I, Baba S et al. Prevalence of risk factors and severity of active trachoma in southern Sudan: an ordinal analysis. Am J Trop Med Hyg. 2007;77(1):126-32.

15. Koizumi IKD, Amaral RKK, Favaro EM, Morimoto WM, Medina NH. Prevalência do tracoma em pré-escolares e escolares no município de São Paulo. Rev Saúde Pública = J Public Health. 2005;39(6):937-42.

16. Oliveira MMMD, Silva JCSL, Vieira JJC, Silva MC, Santos AMC. Prevalência de tracoma em escola de município litorâneo, Estado de Alagoas. Brasil. Arq Bras Oftalmol. 2003;66(4):5. [XXXII Congresso Brasileiro de Oftalmologia, 2003; Salvador, Brasil].

17. Reis ACPP, Chaves C, Cohen JM, Belfort F, Oliveira MP, Junior JB. Detecção de tracoma e doenças corneanas em índios da região do Alto Rio Negro. Arq Bras Oftalmol. 2002;65(1):79-81.
18. Cruz AAV, Medina NH, Gonçalves GFOR, Gomes UA. Distribuição espacial e determinantes sociais do tracoma na cidade de São Gabriel da Cachoeira - AM. Arq Bras Oftalmol. 2001;64(4):5. [XXXI Congresso Brasileiro de Oftalmologia, 2001; São Paulo, Brasil].

19. Dias VG, Pereira CAC, Alves MC, Gonçalves FF, Mantovan FB. Tracoma em instituições públicas municipais de ensino de Presidente Prudente (SP). Arq Bras Oftalmol. 2003;66(4):5. [XXXII Congresso Brasileiro de Oftalmologia, 2003, Salvador, Brasil].

20. Medina NH, Gentil RM, Oliveira MB, Sartori MF, Cabral JH, Vasconcelos MS. Investigação epidemiológica do tracoma em pré-escolares e escolares nos municípios de Franco da Rocha e Francisco Morato. Arq Bras Oftalmol. 1994;57(3):154-8.

21. Nóbrega MJ, Farah ALHL, Miller D, Kang HM, Haddad LH. Estudo clínico e laboratorial do tracoma em escolares de Joinville, Santa Catarina, Brasil. Arq Bras Oftalmol. 1998;61(5):551-6.

22. Carvalho RC, Falcão R, Cohen J, Chaves C, Scarpi MJ. Prevalência de tracoma em escolares de Manaus. Arq Bras Oftalmol. 1997;60(3):243-7.

23. Paula JS, Medina NH, Cruz AA. Trachoma among the Yanomami Indians. Braz J Med Biol Res. 2002;35(10):1153-7.

24. Klauss V, Schaller UC. International initiatives for the prevention of blindness. Ophthalmologe. 2007;104(10):855-9.

25. Astle WF, Wiafe B, Ingram AD, Mwanga M, Glassco CB. Trachoma control in Southern Zambia - an international team project employing the SAFE strategy. Ophthalmic Epidemiol. 2006;13(4):227-36.

26. West SK. Blinding trachoma: prevention with the safe strategy. Am J Trop Med Hyg. 2003;69(5 Suppl):18-23.

27. Medina NH, Luz IMR, Lopes MFC, Vieira LC, Caligaris LSA. Uso da azitromicina para o controle do tracoma em instituição. Arq Bras Oftalmol. 2002;65(4):4. [XV Congresso Brasileiro de Prevenção e Reabilitação Visual; 2002, Curitiba, Brasil]

28. Solomon AW, Harding-Esch E, Alexander ND, Aguirre A, Holland MJ, Bailey RL et al. Two doses of azithromycin to eliminate trachoma in a Tanzanian community. N Engl J Med. 2008; 358(17):1870-1.

29. Edwards T, Harding-Esch EM, Hailu G, Andreason A, Mabey DC, Todd J et al. Risk factors for active trachoma and Chlamydia trachomatis infection in rural Ethiopia after mass treatment with azithromycin. Trop Med Int Health. 2008; 13(4):556-65.

30. West S, Alemayehu W, Munoz B, Gower EW. Azithromycin prevents recurrence of severe trichiasis following trichiasis surgery: STAR trial. Ophthalmic Epidemiol. 2007;14(5):273-7. 